\title{
Commitment Modeling
}

\author{
Frantisek Hunka \\ University of Ostrava, Faculty of \\ Science \\ Dvorakova 7, 70103 Ostrava 1 \\ Czech Republic \\ Email: frantisek.hunka@osu.cz
}

\author{
Jaroslav Zacek \\ University of Ostrava, Faculty of \\ Science \\ Dvorakova 7, 70103 Ostrava 1, \\ Czech Republic \\ Email: jaroslav.zacek@osu.cz
}

\author{
Jiri Matula \\ University of Ostrava, Faculty of \\ Science \\ Dvorakova 7, 70103 Ostrava 1, \\ Czech Republic \\ Email: jiri.matula@osu.cz
}

\begin{abstract}
The notion of commitment is a principal notion in human communication in social systems. It can be also viewed as an elementary contract in which human beings enter into and comply with to achieve their business goals. However, different methodologies use slightly different paradigm to model this notion. The paper firstly analyzes the commitment usage in economic transactions in general, and then investigates the DEMO Enterprise Ontology (Design \& Engineering Methodology for Organizations) and Resource Event Agent (REA) ontology and their capabilities for commitment modeling. Possibilities of these modeling approaches are shown in an example of a practical application. The main asset of the paper is to empirically explore the DEMO's prescriptive and REA's descriptive abilities for enterprise information system design and to reveal their strong and weak points.
\end{abstract}

\section{INTRODUCTION}

$\mathrm{T}$ HE notion of commitment belongs to fundamental notions in the social systems, in which human beings are the core participants, according to [4]. In general, human beings plan and achieve their goals through conversation (coordination). Human beings who are involved in conversation concerning commitment are marked as performer (anyone who expose intention) and addressee (somebody, to which the intention is addressed). We can distinguish an individual commitment, in which the performer exposes intention towards the addressee and paired (coupled) commitments, in which one commitment is in consideration of another 'paired' commitment. Individual commitments are easier elaborated and structured because there is only one commitment over which human beings have their roles. In simple case 'paired commitments' are represented by two commitments in which one commitment is in consideration of the other or it may be represented by two groups of commitments in which one group of commitments is in consideration of the other group.

According to $[1,6]$, an economic commitment is a type of obligation by one human being to transfer economic resources to another human being at some specified point of time usually in future. Commitments can be a part of contract or may stay alone, in which case they are often re- garded as uncontracted commitment. Commitments are also regarded as atomic contracts, according to [5]. In the simplest form of commitment (contract) two contract parties (human beings) commits to an exchange of resources such as goods for money or service for money, i.e. to a pair of transactions of resources from one party to the other. The important thing about it is that one transaction is in consideration of the other.

There are two methodologies, we focus on, dealing with business process modeling and working with the notion of commitment. The DEMO enterprise ontology [3] and the REA value modeling ontology $[2,5,6]$. The DEMO methodology has its foundation in the DEMO enterprise ontology and provides a strong theoretical foundation for business process modeling. On the base of the transaction axiom DEMO has 'integrated' commitment in its transaction pattern. Universal transaction pattern is a very powerful concept, which among others enables working actions in current and in the future in the uniform way. DEMO as a generic methodology works primarily with individual commitment, which is structured in the form of transaction in a tree structures.

The REA value modeling ontology originates from accountancy systems and provides a domain specific platform for value modeling business processes. The term value modeling means that REA approach keeps track of primary and raw data about economic resource values. REA model is composed of at least two transactions of economic resources, where one transaction is in consideration of the other. REA considers service to be an economic resource. Rather than focusing on debits and credits which by design omit important data about economic event, REA proposes capturing the detail about each resource under the organization's control, the events that change the amount of each resource, and the agents who participate in these events, according to $[6,7,9]$. Both the REA and DEMO methodologies utilize the notion of transaction in which commitment is included. Commitment itself is a principal notion in human communication. The other methodologies do not provide such concepts and such ways for modeling. Our motivation is to empirically explore the DEMO's 
prescriptive and REA's descriptive abilities for enterprise information systems design, utilizing commitment modeling, and to reveal their strong and weak points.

The remainder of the paper is organized as follows: Section 2 deals with commitment modeling in DEMO. REA commitment modeling is depicted in section 3. Practical examples of commitment usage are stated in section 4 . The resulting findings are discussed in section 5. The final section contains conclusions and a summary of future work.

\section{DEMO COMMITMEnT Modeling}

According to the DEMO methodology, human beings, of which an organization is composed, perform two kinds of acts: production acts and coordination acts. By performing coordination acts human beings enter into and comply with commitments towards each other regarding the performance of production acts $[3,4]$. Commitments in DEMO are included in the standard transaction pattern as its 'integral' part. The standard transaction is depicted in Fig. 1. Each act is followed by a corresponding fact representing a concrete state. In general, an elementary state of affairs in a world is a fact.

Actually, transaction pattern expresses coordination acts and facts that enclose the production act and fact. When studying the transaction pattern in detail one soon finds out that the proposition phase of the transaction pattern represents a commitment. In this phase the initiator exposes his or her request concerning the production fact and if the executor promises the request, both actors enter into a commitment. Execution phase of the transaction pattern concerns production act and fact. The result phase of the transaction pattern represents the fulfilling of the commitment in case of 'happy path'. However in reality, it often happens that the proposition phase and the execution phase do not follow immediately one after another. Usually, there is a time lag between both phases. The case in which there is a time lag between proposition and execution phases is more frequent and therefore more important for studying than the case when the proposition phase fluently continues into the execution phase and the result phase. As can be seen from Fig. 1, the DEMO methodology puts all phases into one transaction pattern. The time lag between phases makes the transaction pattern closer to reality. The transaction pattern is able to capture activities in which the execution phase and result phase fluently follows after the proposition phase and the case in which there is a time lag between the proposition phase and the execution and result phases. In practice it means that the case when a customer rents a car immediately is the same as the case when a customer makes a reservation of renting a car for the following weekend. Both these seemingly different cases are modelled according to the same transaction pattern.

In DEMO, a business process is formed by transaction(s) structured in a tree arrangement (parent-child relation). It frequently happens that one business process has transactions which the execution and result phases will happen in future and which will affect the other business process. These

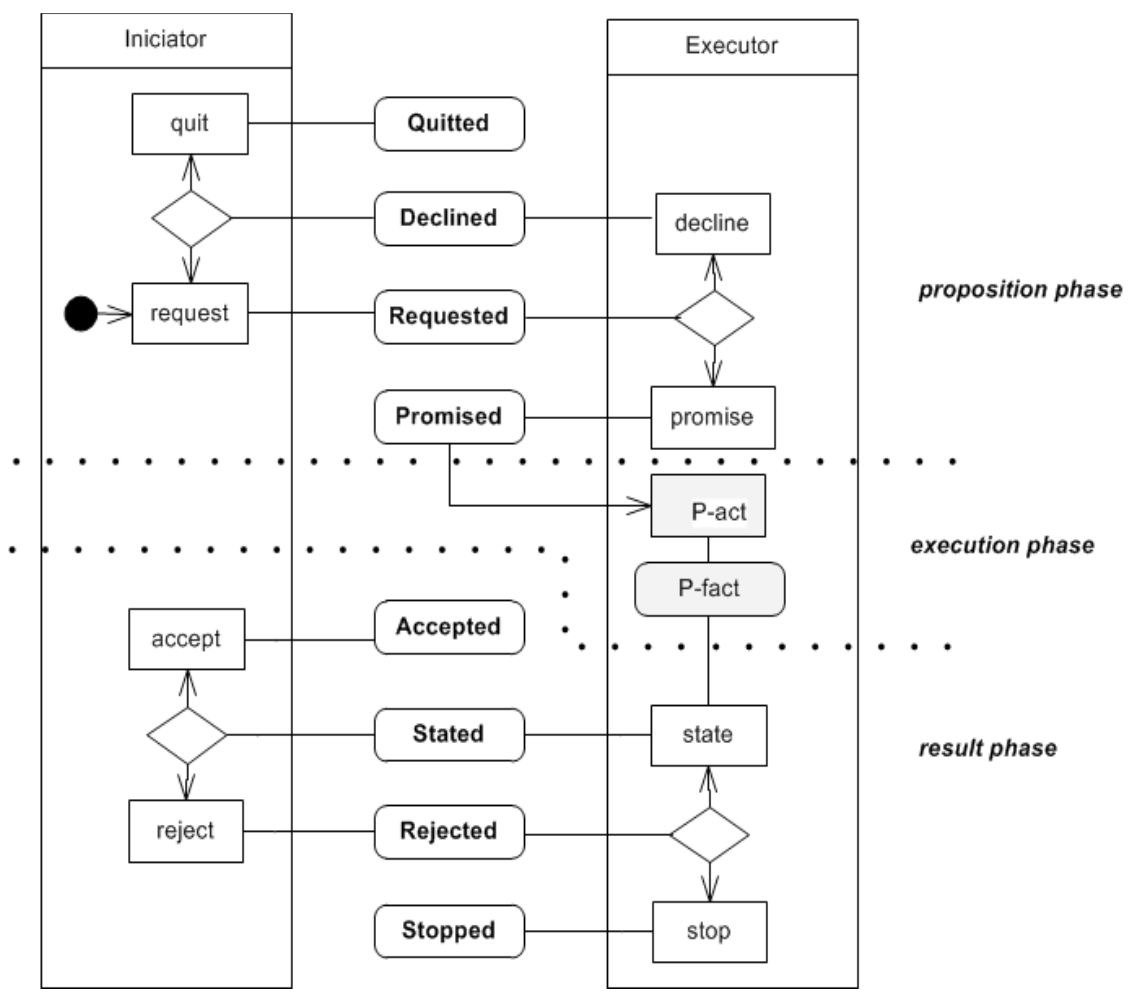

Fig. 1. The Standard Transaction Pattern Source: [3] 
processes have to be loosely coupled through information links.

Figure 1 contains two 'swimlanes', one for the initiator and the other for the executor. Inside these swimlanes are indicated coordination acts which may happen. In the middle, there are shown coordination facts, which are incurred by the acts.

The executor swimlane also contains the production act (P-act) and the production fact (P-fact). The production act and fact are placed in the executor's swimlane because production is exclusively executor's activity. This figure also represents the DEMO state machine in which transitions are drawn within the swimlanes and the states are situated in the middle between the swimlanes. As the DEMO methodology is a generic methodology for business process modeling the DEMO transaction pattern can be considered to be an individual commitment of the corresponding production fact.

It may be concluded that the DEMO methodology enables to model transactions with a time lag between the proposition and the result phases of transaction according to unique transaction pattern. In addition, the DEMO Enterprise Ontology and its models provide prescriptive information system for the enterprise. This information system enforces model compliance. In short, it enforces all actors to comply with the allowed state transitions. This is done by the Transaction axiom one of the four axioms on which the DEMO Enterprise Ontology is based.

\section{REA COMMITMENT Modeling}

Commitment entities and their relationships with other entities are shown in Fig. 2. The commitment entity copies to a considerable extent the structure of the event entity, by which we mean the existence of an increment and decrement commitment and the exchange reciprocity relationship. The exchange reciprocity relationship between the increment and decrement commitments identifies which resources are promised to be exchange for which others $[6,7,8]$.

Each commitment is related to an economic resource by a reservation relationship which specifies which resources will be needed or expected by future economic events. The reservation relationship between the resource and commitment represents the features of the resource and rights associated with the resource that will be changed or transferred by a future economic event. As a matter of fact, each commitment must be related by committed provide and committed receive relationships to economic agents.

The other crucial relationship is the fulfillment relationship which relates a corresponding commitment entity to an event entity. The purpose of this relationship is to validate whether the economic events fulfill corresponding commitments. A REA model represents one business process. REA transactions are paired.

Contrary to the DEMO methodology, REA ontology is a domain specific methodology, which focuses on all information systems where the values of resources are traced. There are three basic differences between the DEMO methodology and the REA modeling approach in terms of

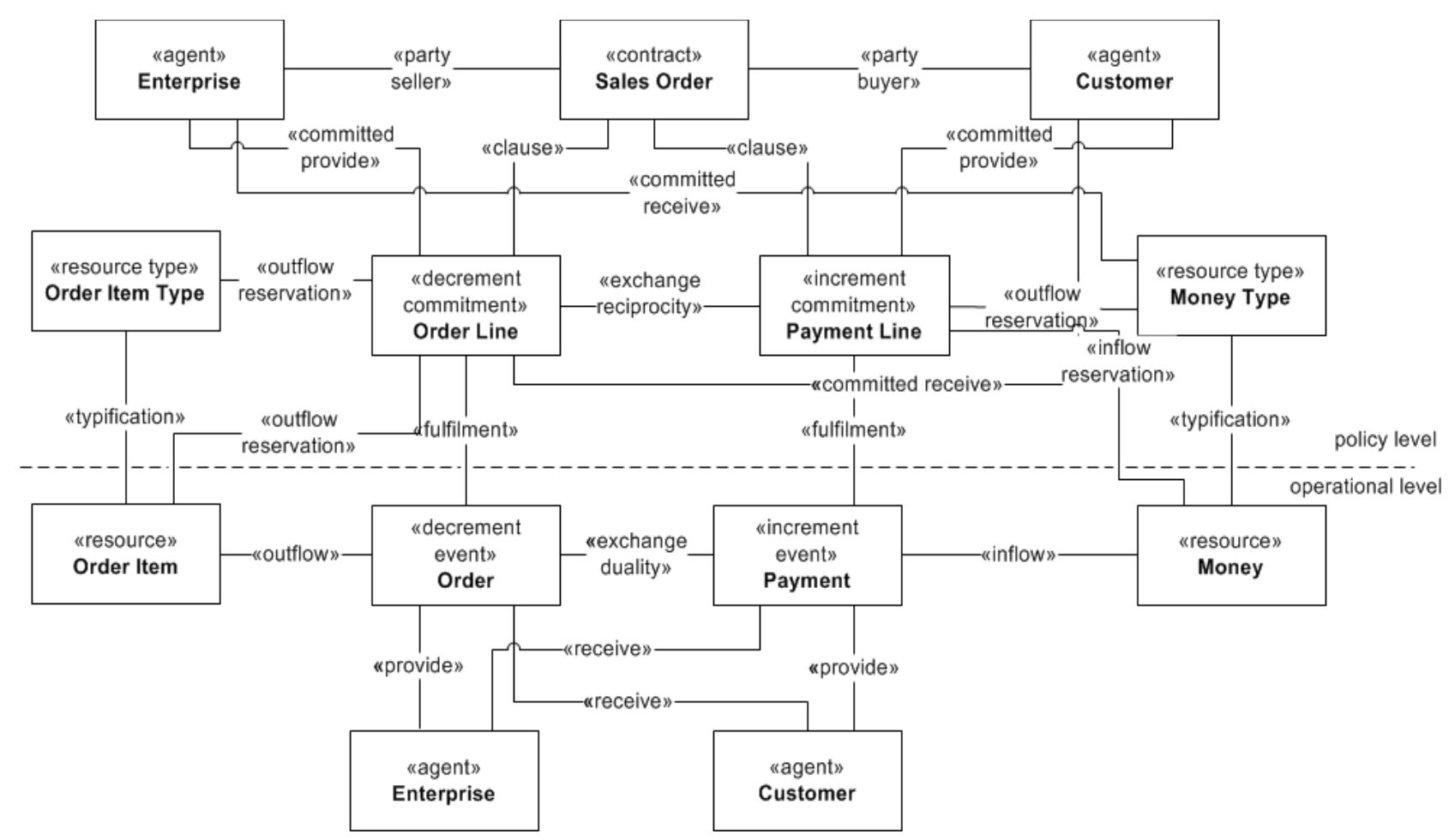

Fig. 2 REA model of sales order Adapted from[6] 
commitment modeling. At first, REA as other business process modeling methodologies works only with production activities and production results. It does not work with communication (coordination). At second, the REA modeling approach distinguishes between current and past economic events and future economic events which are denoted in REA as commitment entities. An economic event itself captures changing of property rights to economic resources or captures consuming, usage, and production of economic resources.

A commitment entity addresses the issue of modeling promises of future economic events and the issue of reservation of resources. The reason for this solution has its origin in accountancy systems from which REA ontology has developed into full-fledged tools for information systems modeling. Economic events specify according REA ontology only actual increment or decrement of resources, not the future increment or decrement of resources. Finally, REA distinguishes two principal transaction kinds, increment transaction, decrement transaction, and these transactions are inherently arranged as paired. The exchange reciprocity relates increment commitments to decrement commitments and the duality relationship relates increment economic events to decrement economic events. The REA modeling approach results in descriptive information systems that are based on exchange, consumption, usage and production of economic resources.

\section{Practical Example of Commitment Usage}

This practical example can elucidate the differences and similarities in a commitment modeling in the context of the above mentioned modeling approaches. The rent-a-car example covers both current and future events and on the other hand, it is not too complex to comprehend [4]. To introduce the problem a short narrative description follows. Rent-a-car is a service which is provided either to walk-in customers or customers who make a rental reservation by telephone, fax or email. A car may be rented on the same day or may be reserved for a specific term in the future after a contract between an employee of the rental company and a customer has been signed. The company which rents out cars has many branches around the country. So, the rented car may be picked up and dropped off at different branches. The rental payment depends directly on the number of days of rental and kind of rented car. Among other things, the signed contrast states the period of the rental and the name of the branch where the car will be dropped off. If the period of rental or/and the drop off branch do not coincide with the conditions in the signed contract, the customer is liable for a penalty payment. The rental payment must be made by the starting day of the rental at the latest. Additional penalty payments must be made at the drop off point.

\section{A. DEMO Modeling Approach}

From the short narrative actor roles and transaction kinds can be identified. There are four actor roles: CA01 - renter, CA02 - driver, A01 - rental starter and A02 - rental ender. The renter actor role and the driver actor role may represent the same or different person. In this example, we can identify six transactions which represent two business processes. The first business process is composed of the following transactions: T01 rental start, T02 rental payment, T03 car pick-up, and T04 car drop-off. The second business process

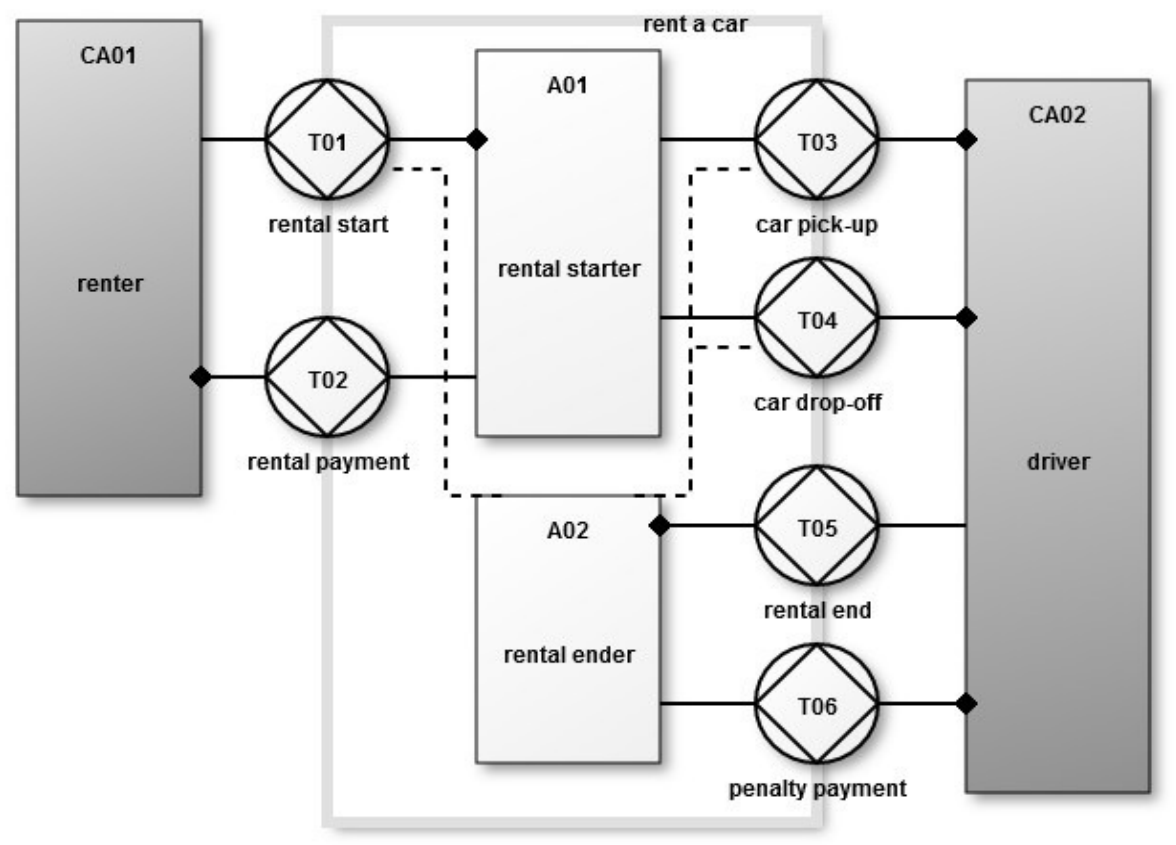

Fig. 3 The DEMO Construction Model 
is constituted of the following transactions: T05 rental end, and T06 penalty payment. For the commitment modeling, the first business process is more illustrative than the second one. The structure of the first business process is as follows. T01 - rental start is a parent transaction and all other three transactions are child transactions (T02, T03, and T04). Conversely, T02 rental payment, T03 car pick-up and T04 car drop-off transactions are enclosed in T01 rental start transaction. Conclusion of T01 rental start transaction also means that the contract between a renter and a rental starter has been signed. The DEMO construction model capturing actor roles and corresponding transactions is depicted in Fig.3.

The renter - CA01 and the driver - CA02 are composite actor roles colored dark-grey. The rental starter - A01 and the rental ender actor roles are elementary actor roles colored white. All six transactions are placed at the interface of the rent-a-car system and environment. The solid lines among a transaction and two different actor roles (each transaction is connected by solid lines exactly with two actor roles) represent initiator and executor. The executor role is indicated by the solid dot at the actor's role.

T01 transaction encloses T02, T03, and T04 transactions. When T01 transaction is completed it means the rental payment transaction (T02) must be paid (completed) and the car pick-up transaction (T03) and the car drop-off transaction (T04) must be promised. It means that when T01 rental start is completed the driver can either pick-up a corresponding car type now or pick-up a corresponding car at some future point in time. More formally, there may/ may not be a time lag between the promise transaction step and the accept transaction step in T03 transaction. In case of T04 transaction, we suppose that there will be a time lag between the promise transaction step and the accept transaction step in the duration of the rental.

However, information about rental start, car pick-up and car drop-off are necessary for the second business process, which is formed by T05 and T06 transactions. According to the above mentioned information, T05 transaction decides whether the driver must pay a penalty or not. The dashed lined in the DEMO construction model between a transaction and an actor role indicates the flow of information. Detailed view on the inside of an individual business process provides the Process Structure Diagram. This Process Structure Diagram of rent-a-car for the first business process is depicted in Fig. 4.

The solid lines in the figure represent causal relations between individual transaction steps. A small box at the edge of the transaction stands for an action (transition) whereas a small circle indicates a fact (state). On the request of the renter for the T01 rental start transaction the rental starter (A01) promises rental start and requests three next transaction kinds, namely, T03 car pick-up transaction, T02 rental payment transaction, and T04 car drop-off transaction. T03 and T04 transactions are functionally similar and the rental starter is satisfied with the promise transaction step in both cases. This means in practice that the driver or delegated renter makes an agreement with the rental starter about the particulars related to the car pick-up and the car drop-off activities. The further view on this state says that

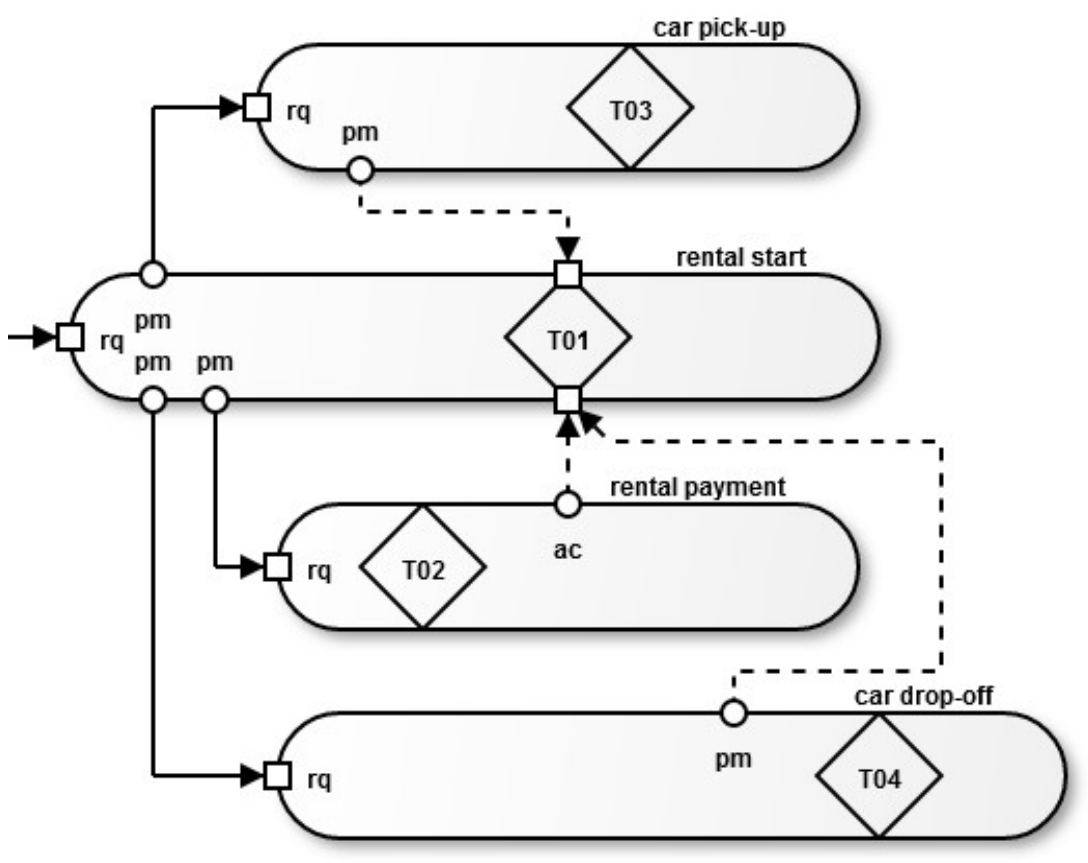

Fig. 4 The DEMO Process Structure Diagram 
this is the only possible way how to solve this issue in which there is evidently a time lag between the promise transaction step and the time when the driver really will pick-up a car at a given branch of the car rent company. The same also holds for the car drop-off transaction. T02 rental payment transaction needed to be completed entirely which means that the rental starter has to accept amount of the rental payment. In terms of time when the rental payment is made, it is essential for the car rental to be paid before a car is being picked up. Figure 4 makes it clear because in case the rental payment is not concluded it implicates that the rental start can't be concluded either. The details of the DEMO models concerning sequence of transaction steps within a given business process are captured in the process structure diagrams.

\section{B. REA MODELING APPROACH}

REA modeling approach represents rather descriptive than prescriptive model. The whole REA exchange model is formed in one business process. Transactions in a REA model are implicitly paired. Those transactions which increase the value of resources in a REA model are paired with transactions that decrease the value of given resources. Assigning the notions of increment and decrement is dependent on the view the REA model is seen. In the practical example, the following commitments and events are identified: Rent a Car commitment, Rent a Car event, Rental Payment commitment, Rental Payment event, Penalty Payment commitment, and Penalty Payment event. As the REA model introduces 'mirroring' each commitment is reflected in a corresponding event. In addition, each commitment is related to corresponding event by the fulfillment relationship.

The commitment entity in its essence represents promise of the future event and is separated from the corresponding event entity. Event entity itself represents an actual exchange or conversion of economic resources. As can be seen, commitment and event entities do not form an integral unit as it is in the case of the DEMO methodology. On the other hand, this separation is a source of many limitations and deficiencies of the REA modeling approach. A crucial role in a REA model plays a contract entity. The contract entity relates to commitment entities and parties which are involved in the REA model.

\section{Discussion}

One of the basic building blocks of the DEMO methodology is a transaction pattern that provides a detail and truthful generic template in which communication between human beings takes place. This pattern is composed

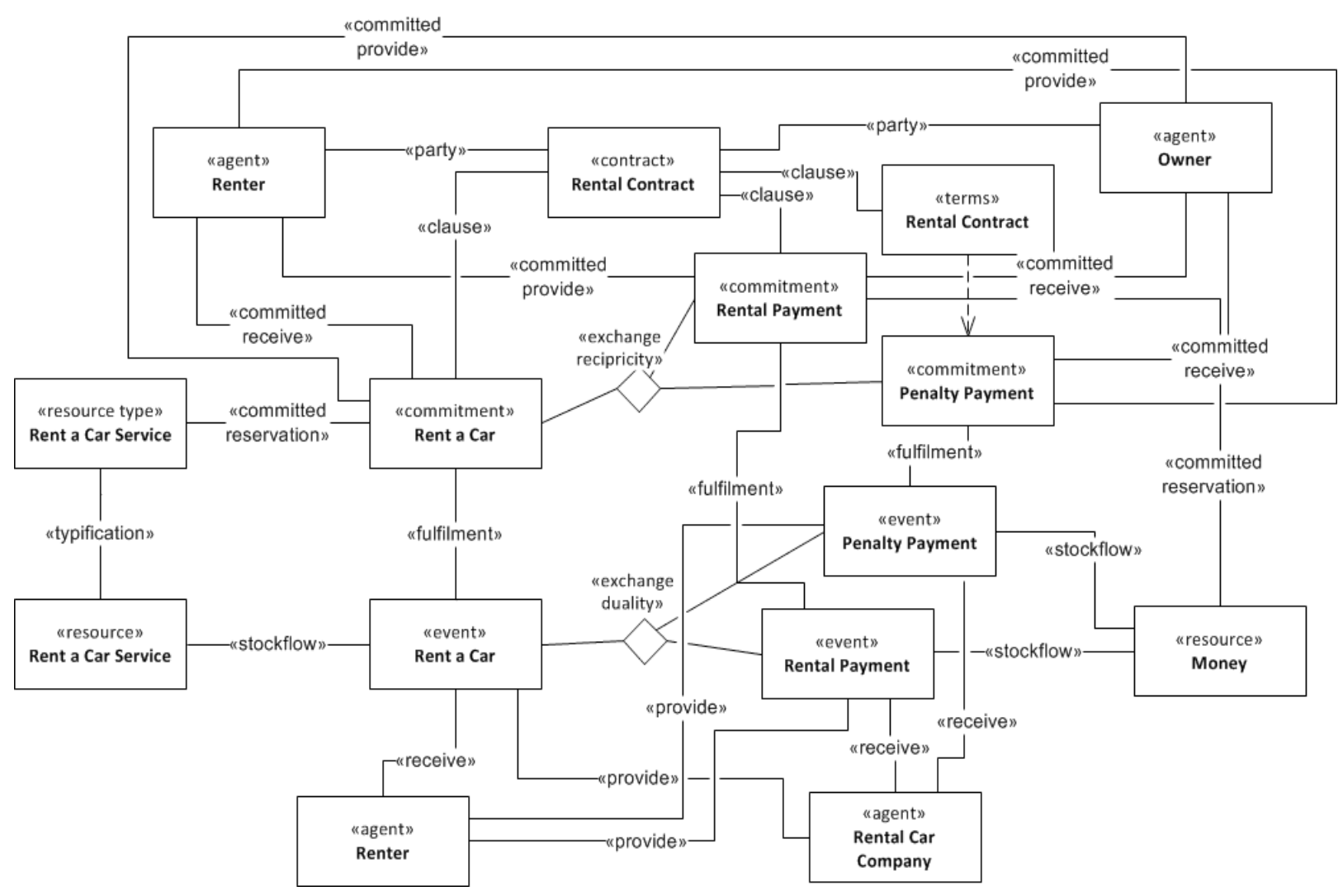

Fig. 5 REA model Source: [7] 
of three phases: the proposition phase, the execution phase and the result phase. The proposition phase represents this part of communication (coordination), in which human beings enter into an agreement concerning the production fact. In short, the proposition phase of the transaction pattern represents a commitment. It can be said that commitment is "integrated 'in this pattern.

The DEMO composition axiom enables DEMO transactions to be arranged in a tree structure. This structure provides a parent-child relationship between transactions. Child transactions are enclosed in the corresponding parent transaction and together form a business process. Business processes may be related to each other by information links (interstriction relationships). In this way, the DEMO methodology is able to capture all the things that happen in reality with great empirical evidence. However, the current DEMO methodology doesn't provide any mechanism supporting the pair transaction arrangement. The parent-child relationship, in which the DEMO transactions are arranged doesn't support this arrangement.

A commitment in REA ontology is modelled as an independent entity that deals with the future economic events and has a relation to economic event(s) that addresses the current and past activities incurred by change of property rights to economic resources or incurred by use, consume, or produce an economic resource.

The REA modeling approach is entirely focused on production activities which are indicated by the corresponding economic events. REA doesn't provide state machine in the sense of the DEMO methodology. Commitment and corresponding economic event are related to each other by the fulfillment relationship which simply indicates whereas the commitment was fulfilled, or not. The core of the REA transaction is formed by a commitment and a corresponding economic event. Two different kinds of the REA transactions are arranged into paired transactions in which one kind of transaction is plan/done in consideration of the other kind of transaction. At the commitment level the paired transactions are related to each other by the reciprocity relationship. At the economic event level the paired transactions are related to each other by the duality relationship. The reciprocity and the duality relationships are crucial relationships in the REA model.

In the practical example, the DEMO construction diagram is formed by six transactions which constitute two business processes. The REA solution represents three mutually paired transactions arranged in one business process (REA model).

\section{Conclusion and Future Research}

The paper presents utilization of the DEMO enterprise ontology and the REA ontology for commitment modeling. The DEMO approach is much precise and consistent. The commitment is inseparable part of the DEMO's transaction pattern. From the practical example and description it follows that the DEMO 'commitment' meets all requirements that are required in reality. However, the current DEMO Enterprise Ontology is not perfectly suitable for capturing an exchange process in which partaking transactions are mutually independent and are not related to each other by the parent-child relationship. This is given by the fact that the DEMO methodology represents a generic ontology in which transactions are arranged in a tree structures. The REA ontology has its origin in accountancy systems and is based on the mechanism of paired transactions. The REA commitment is an independent entity related to other corresponding entities in the REA model. The REA modeling approach is able to work with different transactions as mutually independent transactions with only reciprocity and duality relationships between them which corresponds to the descriptive abilities of REA ontology. Signing and fulfilling a contract is only expressed by the reciprocity and the duality relationships. REA modeling approach doesn't provide the DEMO consistency within its transaction which brings about limited utilization of this methodology.

\section{AcKNOWLEDGEMENT}

The paper was supported by the grant provided by Ministry of Education, Youth and Sports Czech Republic, reference no. SGS15/PRF/2017.

\section{REFERENCES}

[1] G.L. Geerts and W.E. McCarthy, "The Ontological Foundations of REA Enterprise Information Systems". Paper presented at the Annual Meeting of the American Accounting Association, Philadelphia, PA. (2000)

[2] G.L. Geerts and W.E. McCarthy, "Policy-Level Specification in REA Enterprise Information Systems". Journal of Information Systems. Vol 20, No. 2 pp. 37-63. (2006)

[3] J.L.G Dietz, "Enterprise Ontology - Theory and Methodology". Springer-Verlang (2006)

[4] J.L.G. Dietz, "The Essence of Organization. An Introduction to Enterprise Engineering". Sapio bv, 2012.

[5] C.L. Dunn, O.J. Cherrington and A.S. Hollander, "Enterprise Information Systems: A Pattern Based Approach". New York: McGraw-Hill/Irwin (2004)

[6] P. Hruby, "Model-Driven Design Using Business Patterns". SpringerVerlag Berlin Heidelberg (2006)

[7] F. Hunka and R. Belunek, "Transaction Based Business Process Modeling", in Proceedings of the 2015 Federated Conference on Computer Science and Information Systems (FedCSIS). Lodz, 2015, pp. 1397-1402. http://dx.doi.org/10.15439/2015F149.

[8] J. Korczak, M. Hernes and M. Bac, "Fundamental analysis in the multi-agent trading system", in Proceedings of the 2016 Federated Conference on Computer Science and Information Systems (FedCSIS). Gdansk, 2016, pp. 1169--1174. http://dx.doi.org/10.15439/2016F238

[9] P. Sitek, "A declarative decision support framework for supply chain problems", in Proceedings of the 2016 Federated Conference on Computer Science and Information Systems (FedCSIS). Gdansk, 2016, pp. 1215-1222. http://dx.doi.org/10.15439/2016F39 